\title{
SRI LANKAN FEMALE CONSUMERS’ PERCEPTION OF SPORTS BRAS
}

\section{NIROMI SERAM \& ACINI SENARATHNE}

Department of Textile \& Clothing Technology, University of Moratuwa, Katubeddha, Moratuwa, Sri Lanka

With the present global trend of focusing on health-conscious lifestyles, the demand for sportswear has increased markedly. Sports bras form the main category of the women's sportswear apparel industry. This study focuses on understanding the perception of Sri Lankan female consumers regarding sports bras.

This research was conducted based on a sample of young women from the urban population of Sri Lanka. Following the mixed method research technique, a questionnaire survey and several interviews were conducted to collect information on consumer perception and preferences in sports bras.

The findings highlighted that $65.9 \%$ of women were using a sports bra in their day-to-day life, mainly due to the comfort and extra support they provided, while $34.1 \%$ were continuing to use intimate bras even when engaging in physical activities. However, the high prices and difficulty in ensuring a perfect fit were two main reasons that limited the sales of sports bras. It was also noted that consumers prefer to wear a non-wired sports bras and compression sports bras as they provide a perfect fit. They are made of a fabric blended with cotton and Lycra/Spandex and are popular among Sri Lankan female consumers (Lycra and Spandex are different brand names for the same material).

The findings of this survey will prove beneficial to Sri Lankan sport bra designers and manufacturers as this information will guide them to make and supply better products to the Sri Lankan sports bra market.

KEYWORDS: Sports Bras, Consumer, Sri Lanka \& Influence Factors
\end{abstract}

Received: Jan 10, 2018; Accepted: Jan 30, 2018; Published: Mar 02, 2018; Paper Id.: IJBMRAPR20183

\section{INTRODUCTION}

With more and more people adopting active and health-conscious lifestyles, staying fit have become an important aspect of people's busy lifestyles (Lucas, Epstein, \&Kjellstrom, 2014). Consequently, the demand for sportswear/ active wear has increased significantly. A Sports Bra, which is a garment that can be worn in gymnasiums as well as in public places as an outerwear during any kind of physical activity, is one of the main categories of the women's sportswear product line up. This is mainly due to three reasons: viz. Comfort, function, and fashion. So now, many women take to wearing sports bras as everyday bras (Monget, 2016).

Historical data about bras goes back to the $14^{\text {th }}$ century. Starting from the bandeau, the bra has evolved through several stages. As for the sports bra, it was invented in 1977 by Lisa Lindahl and Polly Smith as exercising and fitness became more popular among women, and a need arose from something much more supportive than the regular bra (Hawkins, 2015). The 2000s brought an onslaught of new designs like strapless, one strap, and even a resurgence of corset bras (Hawkins, 2015).

A sports bra is identified as a bra, designed to give extra support and protection to the breasts during strenuous physical activities (Collins, n. d.). Compared with the intimate bra, the sports bra minimizes breast 
movement, reduces discomfort and practically eliminates damage to the chest area.

Whether the cup size is small or large, the wearer needs to select the right sports bra for her based on specific needs such as size, comfort, level of activity that will be performed and the budget. Although a large number of consumers may belong to the same market segment, they do not all behave alike and so a particular product's design and characteristics may not appeal to all the consumers in the same way.

The consumers' perception and behavior are influenced by various socioeconomic and cultural factors (Doublegist, 2013). There are several distinct variables that affect the consumer buying behavior (Doublegist, 2013), such as family influence, peer pressure, age, fashion, educational background, religion, and social status, etc.

The appearance of the garment, which includes color, pattern, color consistency, luster, opacity, hand feel, construction and trimmings will determine the garment's appeal to the consumer(Dedhia \& Gupta, 2009). Besides appearance, performance aspects such as the garment's utility (usefulness) and durability (serviceability), which refers to how well the garment retains its structure and appearance after wear and tear, are also acknowledged as major influences on consumer perception (Dedhia \& Gupta, 2009). However, the garment's basic qualities like the design, materials, construction and finish that determine how well it performs are recognized as the most important elements influencing the consumers' perception (Dedhia \& Gupta, 2009).

Thus, this study focuses on identifying the Sri Lankan female consumers' perception of sports bras.

\section{RESEARCH METHODOLOGY}

The mixed method research approach was adopted, and the data gathered through a questionnaire survey and interviews.

- A questionnaire survey was conducted among the women of 18-35 year age group to learn about their current usage of sports bras.

- Out of the population of Sri Lankan women aged between18 and 35, a sample size of 15 was chosen for the pilot study of the questionnaire. The final questionnaire was fine-tuned after the pilot study. The fullscale questionnaire survey was conducted among 200 women falling within the chosen age category, based on the simple probability sampling technique.

- Interviews were conducted with those women in the 18-35 age group who did not use sports bras during physical activities to pinpoint the reasons for it.

- For the interviews, a purposive sampling technique was adopted and 10 interviews were conducted. Interviews were recorded and transcribed.

\section{DATA ANALYSIS}

136 respondents completed the questionnaires, which were collected for the data analysis. The survey data were analyzed using descriptive statistical analysis techniques. The data from the interviews were analyzed qualitatively. The results obtained were integrated together to conclude the findings. 


\subsection{Overview of the Respondents}

The results show that the vast majority of the respondents belonged to the 18-35 age category. The largest category was the 18-24 years group (51.1\%)with the other age categories being smaller. The results highlighted that $65 \%$ of respondents were single women. $48 \%$ of respondents were at the undergraduate level in their educational path while $45 \%$ worked in the private sector.

\subsection{Involvement in Physical Activities}

Out of the respondents, the majority (24.5\%) participated in sports/gym workouts/ general physical activity once a week, others fortnightly $(23.7 \%)$ or twice or more a week (15.1\%). Reason for this could be that the desire to devote more time to their health and personal matters conflicted with their busy work schedules during the daytime.

According to the results, the majority of the respondents (30.2\%) opted to go for brisk walking. Results show that $27.3 \%$ of respondents played badminton while others engaged in day-to-day activities, yoga, cycling, dancing, running, zumba, volleyball, and Pilates, in descending order of popularity. Results indicate that some of the respondents engaged in two, three or more physical activities.

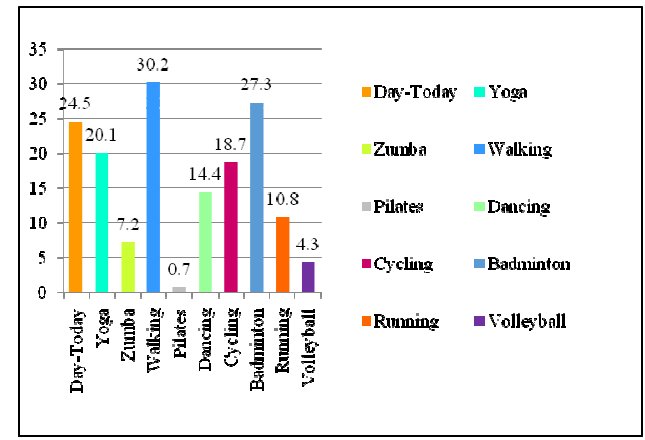

Figure 1: Types of Physical Activities

It was revealed that $47.8 \%$ of the respondents participated in medium impact exercises; the other respondents engaged in low impact or high impact exercises. High impact exercises not only put more stress on the joints, they cause the breasts to bounce more.

\subsection{Usage of Sports Bras During Physical Activities}

With the sports bra trend has really taken off, sportswear is very popular among the women now (Monget, 2016). $65.9 \%$ of women were using a sports bra in their day-to-day life while there were $34.1 \%$ using intimate bras during physical activities. Out of the 139 respondents, most of them were willing to buy a sports bra in the Rs.2000- Rs.5000 price range.

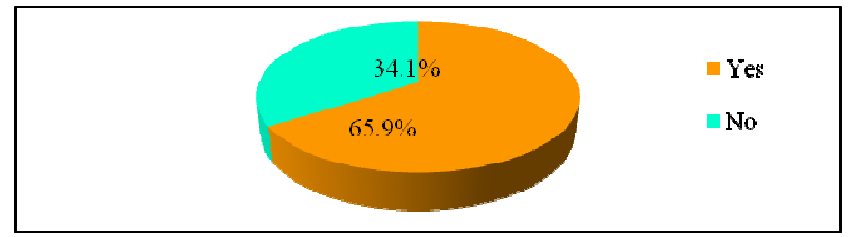

Figure 2: The Usage of Sports Bras 


\subsubsection{Reasons for Choosing a Sports Bra Instead of an Intimate Bra}

When observing the reasons for choosing a sports bra instead of an intimate bra, $77.4 \%$ voted for the comfort that a sports bra offers. Of the sports bra users, $61.3 \%$ asserted that they needed extra support during physical activities, which the intimate bra could not provide. $25.5 \%$ of the respondents thought that their upper body shape improved when they were wearing a sports bra.

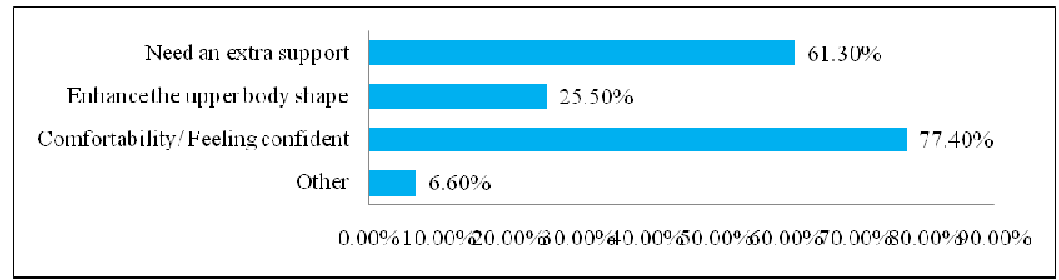

Figure 3: Reasons to Choose a Sports Bra Instead of an Intimate Bra

\subsubsection{Reasons for Not Using a Sports Bra During Sports/Gym/General Physical Activity}

The reasons for not using a sports bra were determined by studying the results of the questionnaire and the interviews.

According to the questionnaire survey, 34.1\% of participants did not use sports bras during sports/gym workouts/general physical activity. They cited various reasons, which can be arranged based on the percentage value of each item.

- Cannot find one that fits.

- $\quad$ Do not need one during their physical activities.

- $\quad$ Cannot find a style that they like.

- Cannot find a comfortable sports bra.

- $\quad$ Do not want to pay more for one.

The reasons for the last three answers could be two-fold; out of the sample population, some respondents did not have the proper bust size required for a sports bra to fit well, while other respondents engaged only occasionally in any kind of physical activity, and not as a habit.

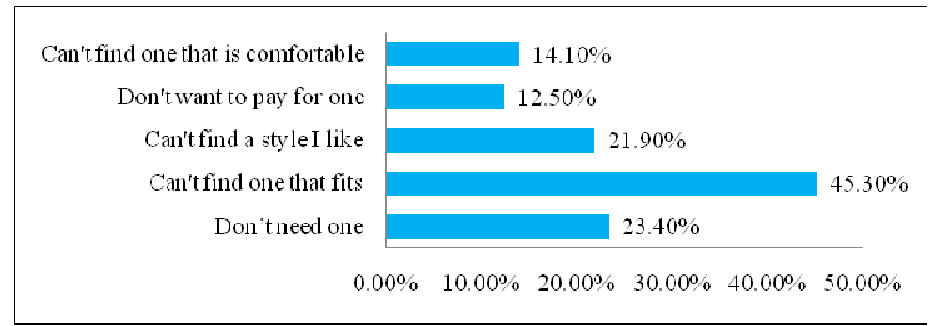

Figure 4: Reasons for Not Using a Sports Bra During Sports/Gym/General Physical Activities

Further, according to the interviews, it became clear that price is one of the significant factors influencing the consumer's buying decision. Finally, after studying all the responses it was possible to list the following points as the 
reasons for not using a sports bra.

- $\quad$ The price of a good sports bra in the Sri Lankan market is really high.

- $\quad$ Feel comfortable with the existing bra which is used for day-to-day activities.

- $\quad$ Size range is not available in the local market to suit small bust sizes.

- Do not have an adequate perception of the sports bra concept.

- $\quad$ Do not understand the finer points of sports bra varieties offered.

\subsection{Consumer Preferences for Sports Bra Features}

Factors such as the color, appearance, embellishments, shape, etc. were rated on a scale based on the level of preference. Then the collected data were quantified to gain an idea about the degree of importance of the above factors. It soon became clear that the majority was giving priority to the comfort and good fit of the sports bra.

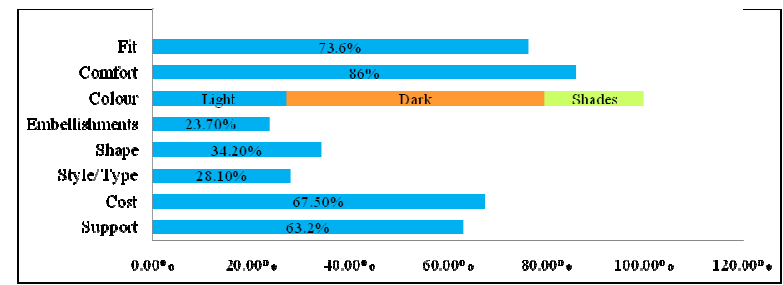

Figure 5: Consumer Preferences for Sports Bra Features

\subsection{Consumer Preferences for Sports Bra Styles}

It was highlighted that most of them (58\%) opted to wear a non-wired sports bras during physical activities. A considerable percentage of respondents (51.8\%) preferred compression sports bras while $37.5 \%$ were in favor of encapsulation sports bras.

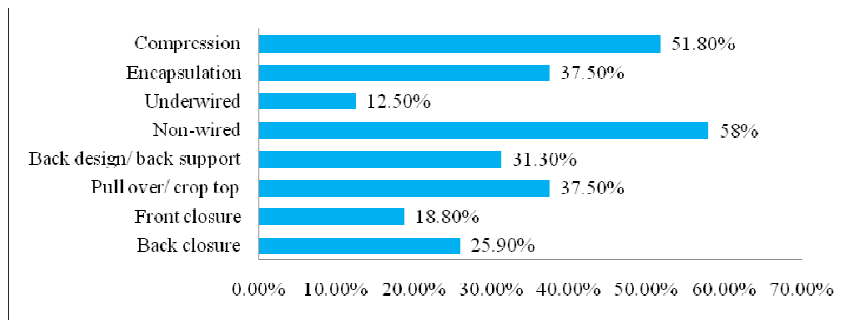

Figure 6: Consumer Preferences for Sports Bra Styles

\subsection{Consumer Preferences for Major Components/ Areas of Sports Bra}

The results show that the cup area is the major component that impacts on a buying decision when shopping for a sports bra. Straps and the bottom band seem to be important areas too. 


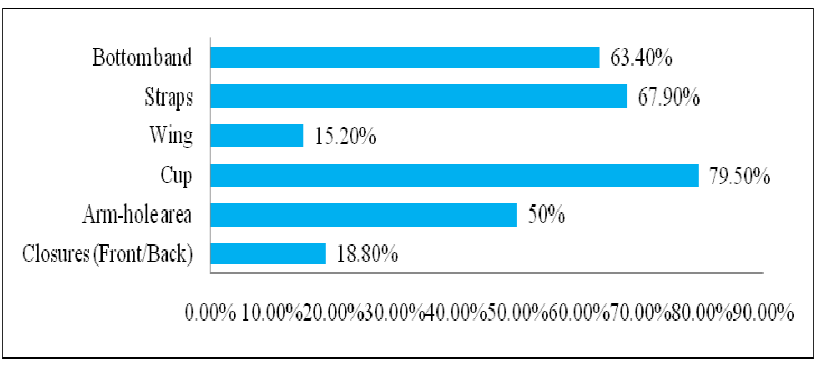

Figure 7: Consumer Preferences for Major Components/ Areas of Sports Bra

\subsection{Consumer Preference for the Fabric}

Results indicated that $45.3 \%$ of women were searching for sports bras made of Cotton and Lycra/ Spandex blended material than other fabric blends. That may be due to the comfort, feel and fit provided by this combination of fabrics and its moisture absorptive properties.

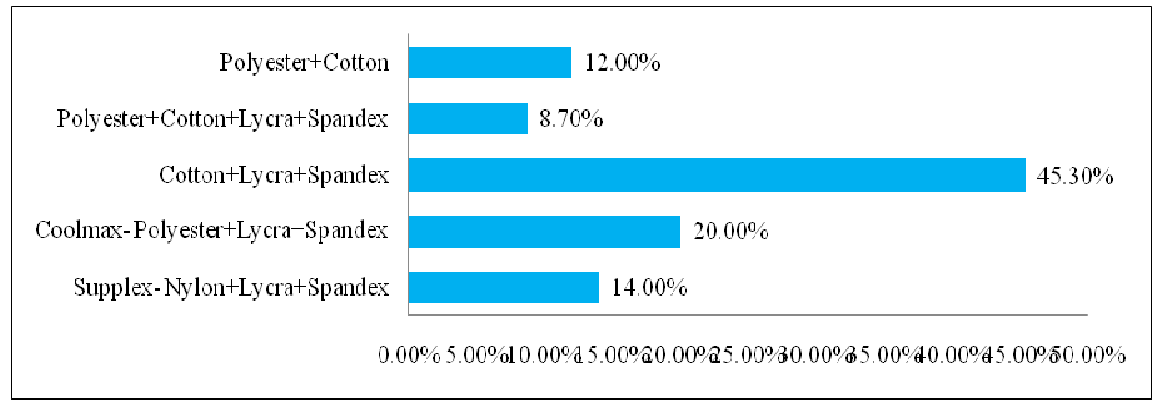

Figure 8: Fabric Preferences

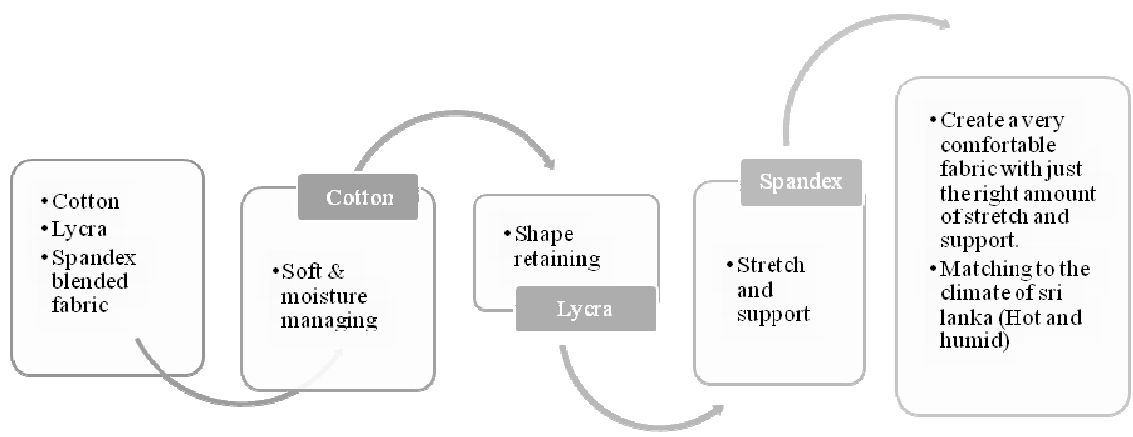

Figure 9: Reasons for the Fabric Selection

\subsection{Consumer Preference of the Brands and the Outlets in the Local Market}

According to the findings, most of the women selected Nike (29\%)as the leading brand for Sports bras and Nike countrywide showrooms $(30 \%)$ as the leading outlet to buy a Sports bra on the local market. It was evident that Nike is a well-known sportswear brand that makes and distributes high-tech, yet simple, robust and wearable sports bra designs to the market. 


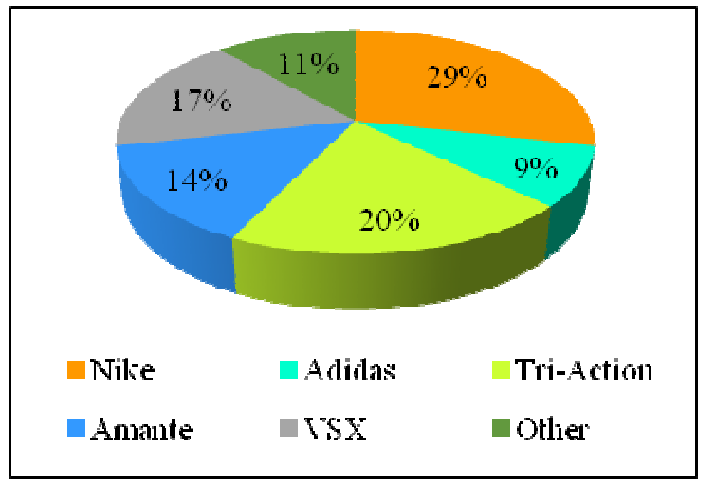

Figure 10: Consumer Preferences for the Sports Bra Brands in the Local Market

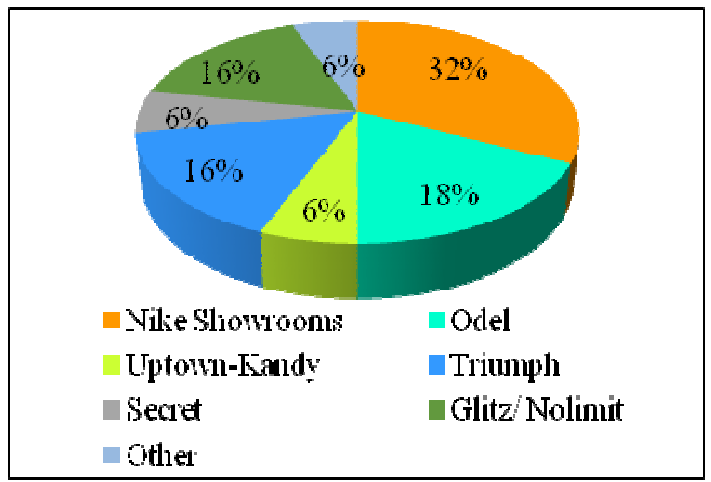

Figure 11: Consumer Preferences for the Sports Bra Outlets in the Local Market

\section{CONCLUSIONS AND RECOMMENDATIONS}

This study focuses on understanding the perception of Sri Lankan female consumers on sports bras. Based on the findings of the questionnaire survey and interviews, it can be concluded that the usage of sports bras is gaining popularity fast. Many young Sri Lankan women have taken to wearing sports bras instead of the intimate bra for medium impact activities, because of the greater comfort, confidence, and support provided by sports bras.

However, some women continue to wear the intimate bra for physical activities, though it does not provide the correct support. The main reasons for not using sports bras can be given as follows:

- $\quad$ Do not understand the finer points of sports bra styles offered.

- $\quad$ The price of a good, branded sports bra in the Sri Lankan market is very high.

- $\quad$ Feel comfortable with the regular bra that is used for day-to-day activities.

- $\quad$ Proper size ranges to fit small made women is not available in the local market.

- Do not have an adequate perception of the sports bra concept.

- $\quad$ Some retailers do not offer cup-size support in their sports bra range.

Further, even though this research was conducted with townsfolk, it was found that most of the consumers do not use the sports bra as an outerwear due to Sri Lanka's unique cultural constraints.

Based on the sampled consumers' expectations, this study would suggest that not only comfortable, well-fitting sports bras that provide medium support be a good option, but also simple, non-wired sports bras would serve well for active Sri Lankan women.

\section{REFERENCES}

1. Burner, T. (n. d.). Sports bras- types, fabrics, what to look for \& more. Me being me - undeniably unapologetically unique and original, Retrieved from: http://taraburner.com/health-wellness/

2. Collins, (n.d.). Definition of sports bra. Collins dictionary, Retrieved from: http://www.collinsdictionary.com/

3. D'Angelo, S. (2011). Your sports bra fitting guide. Fitness magazine, Retrieved from: http://www.fitnessmagazine.com/workout/gear/sports-bras/ 
4. Dedhia, E.\& Gupta, M. (2009). Consumer preferences based on quality and appearance cues, Journal of Textile Association, $\operatorname{vol}(170-$-no. 3)

5. Niromi Seram et al.,, Wearable Technology Products: Awareness in Sri Lankan Market, International Journal of Sales \& Marketing Management Research and Development (IJSMMRD), Volume 6, Issue 3, June - July 2016, pp. 49-58

6. Doublegist (2013). Consumer buying behavior-The influence of culture, DoubleGist. com, Retrieved from: https://www.doublegist.com/consumer-buying-behaviour-influence-culture/

7. Gupta, D. (2011) Functional clothing, -Definition and classification, Indian Journal of Fiber \& Textile Researc (, 36(December), 321-326. DOI: nopr.niscair.res.in/bitstream./13226/IJFTR\% 2036 (4) \%20327-335.pd.

8. Hawkins, A. (2015). The evolution of the bra. Good housekeeping- beauty and fashion, Retrieved from: https:// www.google.lk/ amp/www.goodhousekeeping.com/ beauty/fashion/gmp1291/bra-history/

9. Lucas, R. A., Epstein Y. \& Kjellstrom T. (2014) Excessive occupational heat exposure - a significant ergonomic challenge and health risk for current and future workers. Extreme Physiology \& Medicine, 3(1), 1-8. DOI: 10.1186/2046-7648-3-14.

10. Monget, K. (2016). The sports bra on the rise. Curvelasvegas, Retrieved from:https://www.curvexpo.com/the-sports-brabusiness-on-the-risel

11. Yu, W. \& Zhou, J. (2016). Sports bras and breast kinetics. Advances in Women's Intimate Apparel, 135-140. Retrieved from: http://scitechconnect.elsevier.com/wp-content/uploads/2016/09/main-24.pdf 